\title{
Status of NICA
}

\author{
Vladimir Kekelidze ${ }^{1, a}$, Alexander Kovalenko ${ }^{1}$, Richard Lednicky ${ }^{1}$, Victor Matveev ${ }^{1}$, \\ Igor Meshkov $^{1}$, Alexander Sorin ${ }^{1}$, Grigori Trubnikov ${ }^{1}$ \\ (for NICA Collaboration) \\ ${ }^{1}$ Joint Institute for Nuclear Research, Dubna, 141980 Russia
}

\begin{abstract}
The NICA (Nuclotron-based Ion Collider fAcility) is the new international research facility under construction at the Joint Institute for Nuclear Research (JINR) in Dubna. The main targets of the facility are the following: 1) study of hot and dense baryonic matter at the energy range of the maximum baryonic density; 2) investigation of nucleon spin structure and polarization phenomena; 3 ) development of JINR accelerator facility for high energy physics research based on the new collider of relativistic ions from protons to gold and polarized protons and deuterons as well with the maximum collision energy of $\operatorname{sqrt}\left(\mathrm{s}_{\mathrm{NN}}\right) \sim 11 \mathrm{GeV}\left(\mathrm{Au}^{79+}+\mathrm{Au}^{79+}\right)$ and $\sim$ $27 \mathrm{GeV}(\mathrm{p}+\mathrm{p})$. Two collider detector setups MPD and SPD are foreseen. The setup BM@N (Baryonic Matter at Nuclotron) is commissioned for data taken at the existing Nuclotron beam fixed target area. The MPD construction is in progress whereas the SPD is still at the beginning design stage. An average luminosity of the collider is expected at the level of $10^{27}$ $\mathrm{cm}^{-2} \mathrm{~s}^{-1}$ for $\mathrm{Au}(79+)$ and $10^{32} \mathrm{~cm}^{-2} \mathrm{~s}^{-1}$ for polarized protons at $27 \mathrm{GeV}$. The status of NICA design and construction work is briefly described below.
\end{abstract}

\section{Introduction}

The new research facility NICA aimed at study of heavy ion and polarized proton and deuteron collisions is under design and construction at the Joint Institute for Nuclear Research (JINR) since 2010 [1]. The study of hot and dense baryonic matter should shed light on: in-medium properties of hadrons and the nuclear matter equation of state (EOS); the onset of deconfinement (OD) and/or chiral symmetry restoration (CSR); phase transition (PT), mixed phase and the critical end-point (CEP); possible local parity violation in strong interactions (LPV) [2-5]. It was indicated that heavy ion collisions at ${\sqrt{\mathbf{S}_{\mathrm{NN}}}} 11$ $\mathrm{GeV}$ energy region allow attaining the highest baryon density. Thus, the NICA research domain is attractive as possible domain of phase transitions searching. The study of the nucleon spin content and polarization phenomena in the collisions of polarized protons and light polarized ions introduce a target of the further research at NICA. General scheme of the complex is presented in Fig. 1.

The functional possibilities of planned regimes are illustrated in Fig. 2. NICA is multifunctional facility providing different operating modes and wide spectra of possible projectiles and target combinations.

aemail: Vladimir.Kekelidze@cern.ch 


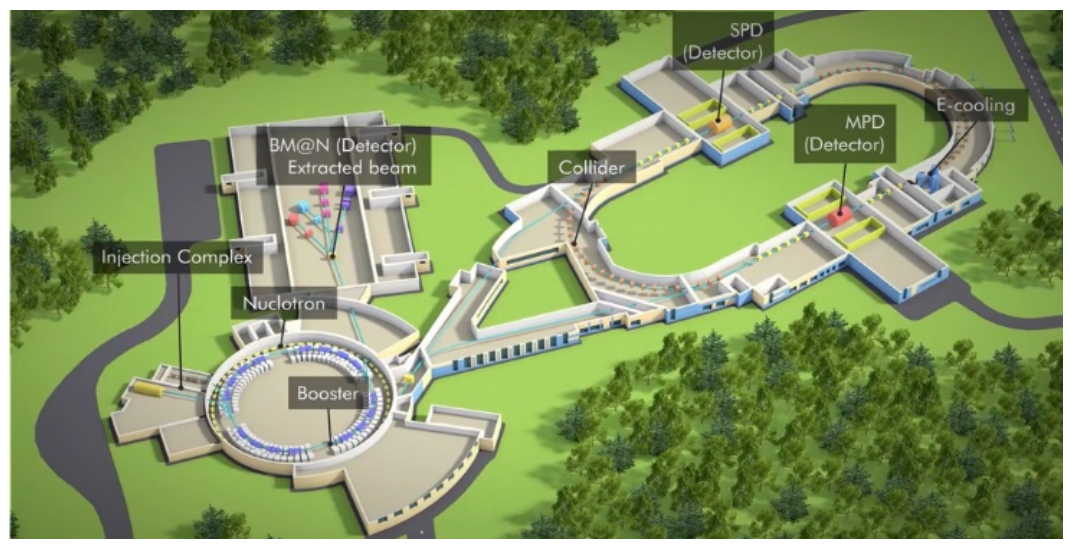

Figure 1. General scheme of the NICA at JINR.

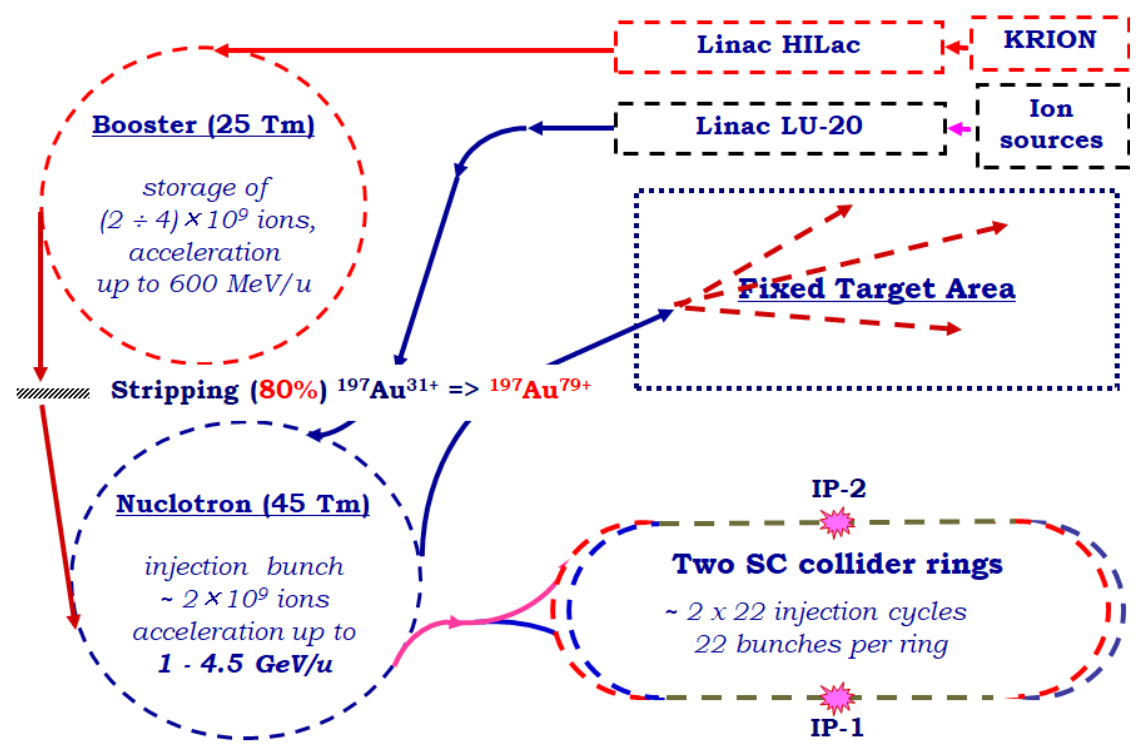

Figure 2. NICA functional scheme providing the specified operation modes for ion and polarized proton and deuteron collisions.

The NICA construction is progressing step-by-step foresees an essential development of the accelerator facility and construction of the new spectrometer BM@N in order to start the fixed target experiments with heavy ion beams extracted from modernized Nuclotron in 2017 and to prepare the MPD start version for commissioning by 2021. Status report on the NICA design and construction is presented regularly at different international meetings and conferences including the ICNFP serial [6], thus we limited this paper by brief description of some new results achieved in a period from 2016 to August 2017.

\section{The accelerator facility}

Four types of ion sources are necessary to cover the particle species spectra aimed for NICA operation modes. These are: 1) the KRION6T- high pulse current, high charge state heavy ion source; 2) laser- based light ion source; 3) the SPI - polarized proton and 
deuteron short pulse, high current source and 4) duoplasmatron type proton and deuteron source for the experiments in unpolarised proton and deuteron mode. Each of the new sources have been commissioned during the past two years. The obtained beam intensities were adequate for NICA specification. An impressive new result -first acceleration of polarized proton beam in the Nuclotron. The beam energy was limited to $2 \mathrm{GeV}$ (below the predicted dangerous spin resonance). Polarization of coasting beam was measured at energy of $270 \mathrm{MeV}$ by means of polarimeter based on internal target and two - arm particle spectrometer. Extrapolation of the measured data on the asymmetry of elastic pp-scattering at the target to beam polarization degree at different points of injection channel, taking all spin rotating elements into account, gave a level of $90 \%$ beam polarization at the source output [7]. Commissioning of the new heavy ion linac (HILAC) and the new front end part of the old linac LU-20 was completed also.

The Nuclotron total running time in 2015-2017 was at the level of 3000 hours. Beams of deuterons, lithium and carbon ions, and polarized deuterons as well, were used mainly for the tasks connected directly with NICA program realization, i.e. commissioning the BM@N setup, tests of the MPD detector elements and data taken for current physics projects $[8,9]$.

The main efforts during the coming two years will be concentrated at completion of the booster system manufacturing, installation, assembling and commissioning. The booster is superconducting strong focusing ion synchrotron $210.96 \mathrm{~m}$ perimeter, variable magnetic rigidity from $1 / 6$ to $25.0 \mathrm{Tm}$ aimed at accelerating different ions over a wide range of charge-to-mass ratio $(\mathrm{q} / \mathrm{A}>0.16)$. The essential feature of the booster operation is low injection energy (3.2 MeV per nucleon) defined by the HILAC parameters. Due to that condition it is necessary to provide proper vacuum level inside the beam chamber (not worse than $1 \times 10^{-11}$ Torr. The peak output energy for $\mathrm{Au}^{+31}$ ions $(0.6 \mathrm{GeV}$ per nucleon) is reach at dipole magnetic field of 1.8 T.The booster magnetic ring will be placed inside the window of the Synchrophasotron yoke. The "tunnel" for the magnets installation is practically prepared except of the straight sections connected the quadrants (arcs of the orbit). Cross section of the booster tunnel with Nuclotron dipole magnet unit is shown in Fig. 3 (left part), whereas a booster dipole preparation for the tests is shown at the right part.
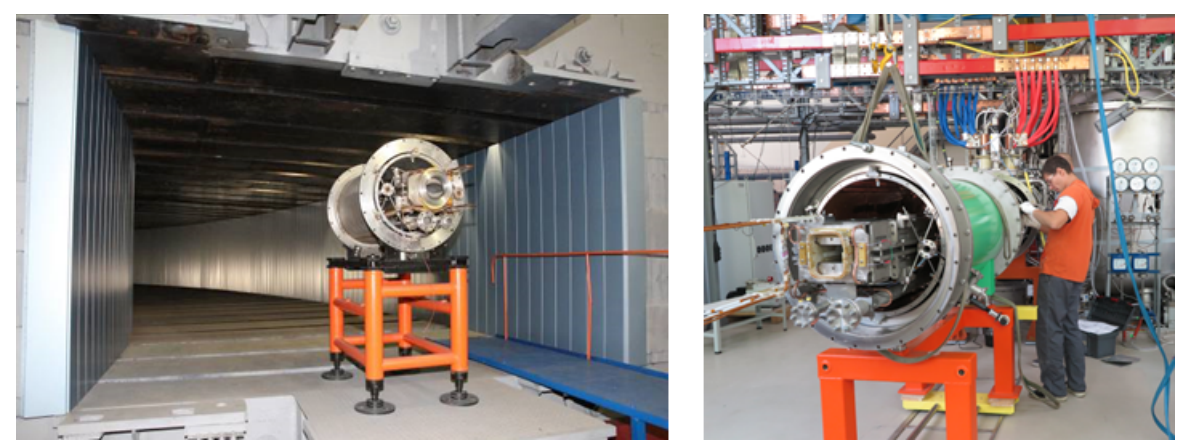

Figure 3. NICA booster "tunnel" and Nuclotron dipole magnet for scale (left photo); the booster dipole preparing for tests at test bench (right photo).

Manufacturing and tests of the booster magnetic system elements is in progress, namely: dipole units are close to completion, yokes and coils for quadrupoles are ready for the assembling. We have some delay with the correcting multipoles and steerers fabrication. The RF and beam electron cooling systems designed and manufactured at the Budker 
Nuclear Physics Institute (BINP, Novosibirsk) have been delivered to Dubna and are ready for installation.

The NICA collider consists of two storage rings with two interaction points (IP). Major parameters of the collider are the following: magnetic rigidity $B \rho=45 \mathrm{Tm}$; vacuum in a beam chamber $-10^{-11}$ Torr; maximum dipole field $2 \mathrm{~T}$; ion kinetic energy range from 1 $\mathrm{GeV} / \mathrm{u}$ to $4.5 \mathrm{GeV} / \mathrm{u}$ for $\mathrm{Au}^{79+}$; zero beam crossing angle at IP; $9 \mathrm{~m}$ space for detector allocations at IP's; average luminosity $L=10^{27} \mathrm{~cm}^{-2} \mathrm{~s}^{-1}$ for gold ion collisions at ${\sqrt{\mathrm{S}_{\mathrm{NN}}}}_{\mathrm{N}}$ 9 $\mathrm{GeV}$ [10]. The collider ring $503.04 \mathrm{~m}$ long (twice as large as the Nuclotron ring) has a racetrack shape and is based on double-aperture (top-to-bottom) superferric magnets dipoles and quadrupoles. A superconducting NbTi composite hollow-tube cable for the magnets is designed and manufactured at the Laboratory. The first set of full size prototype magnets has been produced and passed all necessary tests. The obtained data of the magnetic field measurements have demonstrated the field distribution acceptable for NICA heavy ion operation mode. Serial fabrication of the magnets has to be started in 2018 .

The NICA cryogenics will be based on the modernized liquid helium plant that was built in the early 1990s for the Nuclotron. The main goals of the modernization are: increasing of the total refrigerator power from 4000 to $8000 \mathrm{~W}$ at $4.5 \mathrm{~K}$, making a new distribution system of liquid helium and ensuring the shortest possible cooldown time. These goals will be achieved by means of an additional $1000 \mathrm{l} / \mathrm{h}$ helium liquefier and satellite refrigerators located near the accelerator rings, a nitrogen system that will be used for magnet thermal shield cooling at $77 \mathrm{~K}$ and at the first stage of cooling down all three rings of the Nuclotron/NICA with the total length of about $1 \mathrm{~km}$ and cold mass of 290 tons. Necessary equipment had been ordered and delivered partially. Work is progressing in accordance with the schedule.

\section{Physics program at NICA}

Basic physics objective that NICA research can contribute to are the following: 1) bulk properties, EOS - particle yields \& spectra, ratios, femtoscopy, flow; 2) in-medium modification of hadron properties -onset of low-mass dilepton enhancement; 3) deconfinement (chiral) phase transition at high baryon density - enhanced strangeness production; 4) QCD critical point - event-by-event fluctuations \& correlations;5) chiral magnetic (vortical) effect, $\Lambda$-polarization; 6) $\gamma \mathrm{N}$ interactions in dense nuclear matterhypernuclei. NICA domain at QCD PD is shown in Fig. 4. The domain corresponds to the density frontier.
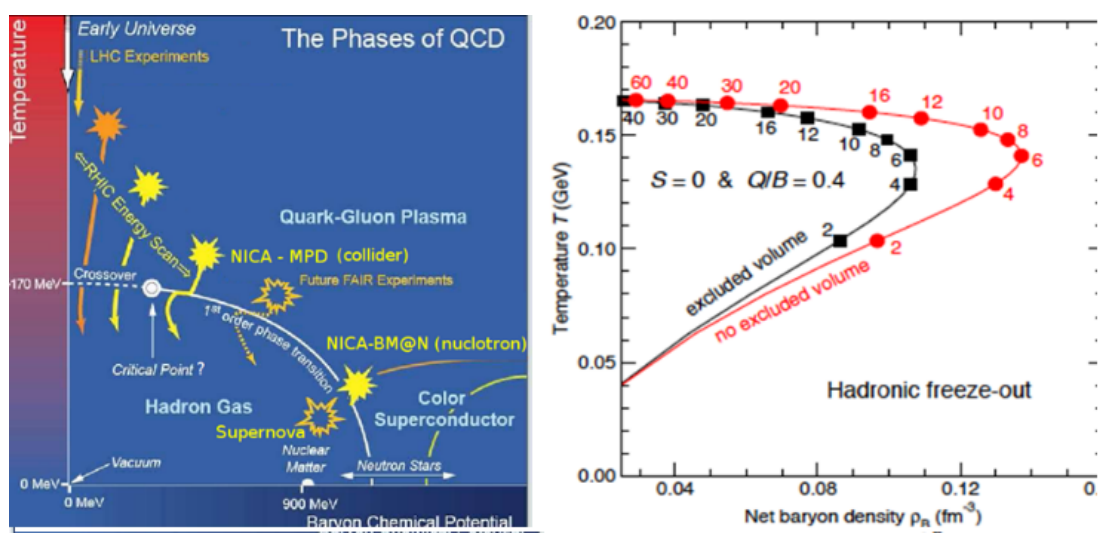

Figure 4. NICA domain at QCD diagram. 
NICA "White Book" was enriched substantially by the new proposals presented at the SQM2016 Conference held in Dubna. The number of groups and organizations interested in heavy ion interactions in the energy domain of $\operatorname{sqrt}\left(\mathrm{s}_{\mathrm{NN}}\right) \sim 4-11 \mathrm{GeV}$ are increased.

\section{The BM@N experiment}

The goals of BM@N setup have been assembled at extracted Nuclotron beam channel are the following:

1. Study of the properties of dense nuclear (dominantly baryonic) matter with strangeness in heavy ion $(\mathrm{A}+\mathrm{A})$ collisions in particular:

- production mechanisms and modifications of hadron properties in dense nuclear matter ("in-medium effects") using different probes, namely: strange mesons, strange and multi-strange baryons; vector mesons via hadronic or dilepton/photon mode).

- study of the EoS with strangeness;

- hyper-matter production: search for light hypernuclei and multi-strange metastable objects.

2. Study of elementary reactions: $p p, p n(d)$ as "reference" to pin down nuclear effects

3. Search for cold" nuclear matter in proton-nuclei collisions.

Start version of BM@N is prepared for data taken at the beam. Acceleration of argon and krypton ions at the Nuclotron up to an energy above $3.5 \mathrm{GeV} / \mathrm{u}$ is scheduled for November 2017 - March 2018.
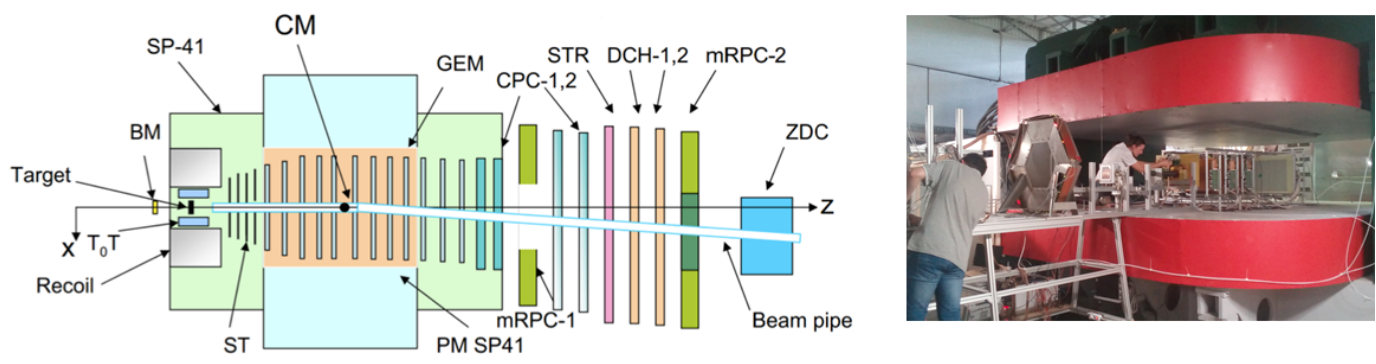

Figure 5. The BM@N setup: GEM - central tracker inside analysing magnet; DCH+Straw - outer tracker behind the magnet; $\mathrm{mRPC}+\mathrm{T} 0$ - time-of-flight system; ZDC - centrality measurements.

\section{The MPD status}

The MPD experimental program is aimed at investigating both hot and dense baryonic matter and some polarization phenomena. Preliminary list of the first priority physics tasks to be performed includes:

1) measurement of a large variety of signals at systematically changing conditions of collision (energy, centrality, system size) using as bulk observables $4 \pi$ geometry particle yields (OD, EOS); multi-strange hyperon yields and spectra (OD, EOS); electromagnetic probes (CSR, OD); azimuthal charged-particle correlations (LPV); event-by-event fluctuation in hadron productions (CEP); correlations involving $\pi, \mathrm{K}, \mathrm{p}, \Lambda$ (OD); directed and elliptic flows for identified hadron species (EOS,OD); reference data (i.e. $p+p)$ will be taken at the same experimental conditions;

2) study of hyperon polarization and other polarization phenomena including possible study of the nucleon spin structure via the Drell-Yan (DY) processes after the MPD upgrade. 
The MPD is a typical collider detector based on a superconducting solenoid. General view of the detector is presented in Fig.6.

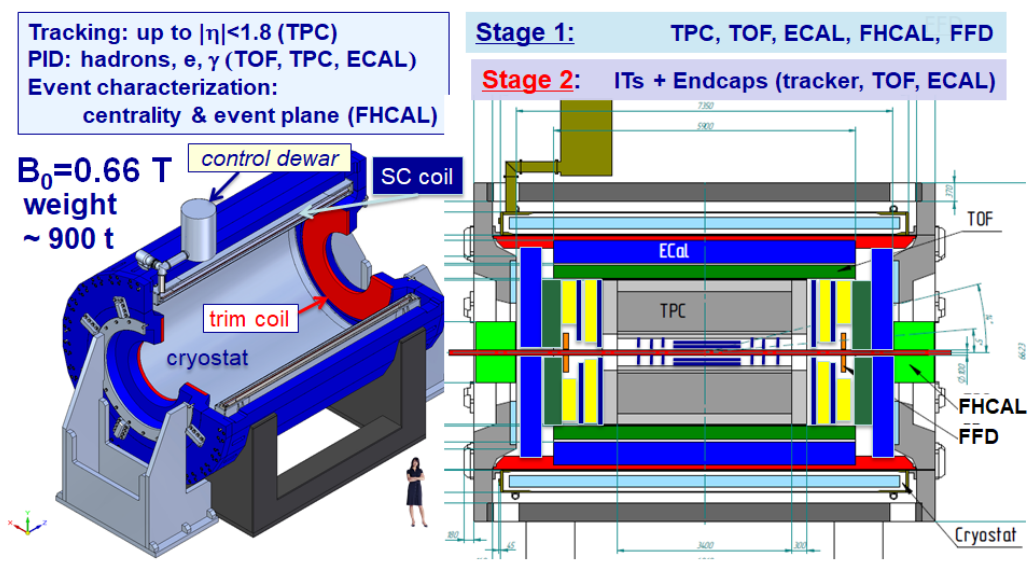

Figure 6. General view of the MPD setup.

Physics program at MPD have been discussed elsewhere. Information about recently published works are presented at NICA site [11]. Status of the setup preparation: SCsolenoid and heavy metallic support parts under manufacturing whereas the first turn detector elements and systems are at preparation to mass production stage. General contractor for the SC solenoid is ASG Superconductors Co. (Genova, Italy).

\section{The SPD status}

SPD detector will be designed and installed in the IP-2 to study spin phenomena. There is a number of processes which could be studied with this detector and with the fixed target detectors at beams extracted from the upgraded Nuclotron, namely: DY processes with longitudinally and transversally polarized $\mathrm{p}$ and $\mathrm{d}$ beams; extraction of unknown (poorly known) parton distribution functions (PDF); PDFs from $\mathrm{J} / \psi$ production processes; spin effects in various exclusive and inclusive reactions; cross sections of diffractive processes; helicity amplitudes and double spin asymmetries (Krisch effect) in elastic reactions; spectroscopy of quarkoniums with any available decay modes. This can be done in the kinematic energy region not available for other experiments. The formation of the collaboration has been started. The analysis of the accelerator issues related to polarized proton and deuteron beams has been progressing during the last three years. Feasible schemes of the polarization control in the Nuclotron and collider have been proposed $[12,13]$. Preparation of SPD CDR is scheduled for 2018.

\section{Civil construction and outlook}

The civil construction of the collider complex is going on in accordance with the plans. the MPD hall should be ready by the summer of 2018, and the entire complex is expected to be completed in 2019.Large scale work on concrete piles pressing into the ground is continuing permanently. More than 4000 of them have been installed. One more urgent task to provide concrete work for stable base formation of the collider and detector setups. 


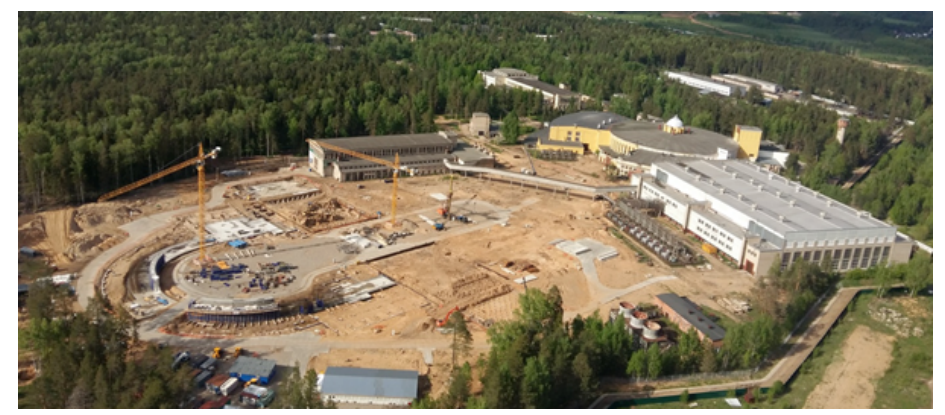

Figure 7. View at NICA construction place in August 2017.

The NICA accelerator and experimental complex and the corresponding research program will provide relevant research, which is both competitive and complementary to ones being carried out at other world leading centers and facilities. Construction of the NICA Complex supported by special grant of the Government of the Russian Federation.

\section{References}

1. V.D.Kekelidze et al., PoS (ICHEP2012) 411, (2013).

2. A.Sissakian et al. Part.\& Nucl Lett. 5, 1 (2008).

3. M.Gazdzicki et al. PL., B585, 115 (2004) .

4. J. Cleymans, and J. Randrup, Phys. Rev. C74 (2006) 04791.

5. B.Mohanty, J. Phys. G: Nucl.\& Part. Phys. 38 (2011) 124023.

6. V.Kekelidze et al. ICNFP2015, EPJ WoC, v.160, p 02015,(2016)

7. V.P.Ladygin et al. arXiv:1711.02850 [physics.ins-det].

8. A.V. Butenko et al. JACoW-IPAC2017-TUPVA116, Conf. Proc.

9. A.V.Butenko et al.JACoW-IPAC2017-TUPVA117 Conf.Proc.

10. G.V.Trubnikov et al . Conf., p.TUPRO005 Proceedings IPAC14.

11. http://www.nica.jinr.ru

12. A.D.Kovalenko et al. 21st High Energy Spin Physics Conf., (2016), UrbanaChampaigne, USA.

13. Yu.N.Filatoff et al.JACoW-IPAC2017-TUPVA112 